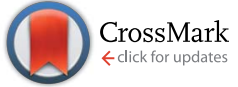

Cite this: J. Mater. Chem. C, 2015, 3, 1537

Received 14th November 2014 Accepted 12th December 2014

DOI: $10.1039 / \mathrm{c} 4 \mathrm{tc} 02602 \mathrm{k}$

www.rsc.org/MaterialsC

\title{
Tuning the photonic properties of chiral nematic mesoporous organosilica with hydrogen-bonded liquid-crystalline assemblies $\uparrow$
}

\author{
Michael Giese, ${ }^{\star a b}$ Tim Krappitz, ${ }^{\text {bc }}$ Ronald Y. Dong, ${ }^{d}$ Carl A. Michal, ${ }^{d}$ \\ Wadood Y. Hamad, ${ }^{e}$ Brian O. Patrick ${ }^{b}$ and Mark J. MacLachlan ${ }^{\star b}$
}

\begin{abstract}
A series of novel hydrogen-bonded assemblies was synthesized and characterized with respect to their liquid-crystalline behaviour. Solid-state NMR spectroscopy gave insight into the columnar nematic mesophase and the corresponding ordering and alignment. Infiltrating the pores of chiral nematic mesoporous organosilica films with the liquid-crystalline compounds gives composite samples that undergo reversible phase changes with temperature, leading to tuneable photonic properties. The unique combination of liquid crystallinity arising from supramolecular interactions (hydrogen bonding) and chiral nematic organisation in a solid-state host is a promising new concept for developing optical sensors.
\end{abstract}

\section{Introduction}

Self-assembly within a confined space is a promising approach for new inventions in nanotechnology and nanoscience as it combines top-down and bottom-up processes. ${ }^{\mathbf{1 , 2}}$ Consequently, many researchers have investigated the changes in physical properties that occur when guests, such as block copolymers, ${ }^{3}$ biomolecules, ${ }^{4}$ nanoparticles ${ }^{5}$ and liquid crystals, ${ }^{6}$ are confined inside a porous host. One example illustrating the influence of confinement effects is the alignment of discotic liquid crystals. Normally, they preferentially align parallel to the surface on which they are deposited, but trapping discotic liquid crystals between two surfaces can lead to reassembly perpendicular to both surfaces, leading to a texture with low birefringence observed by polarizing optical microscopy (POM). ${ }^{2,7}$ In other cases, the physical properties of the guest are affected, sometimes drastically. For example, affected properties include the glass transition and dynamics of polymers ${ }^{\mathbf{1 , 5}, \mathbf{8}}$ or the melting and freezing points of simple fluids. ${ }^{9}$

${ }^{a}$ Institute of Organic Chemistry, University of Duisburg Essen, Universitätsstraße 7, D-45117 Essen, Germany. E-mail: Michael.giese@uni-due.de

${ }^{b}$ Department of Chemistry, University of British Columbia, 2036 Main Mall, Vancouver, BC, V6T1Z1 Canada.E-mail: mmaclach@chem.ubc.ca

'Institute of Organic Chemistry, RWTH Aachen University, Landoltweg 1, D-52074 Aachen, Germany

${ }^{d}$ Department of Physics and Astronomy, University of British Columbia, 6224 Agricultural Road, Vancouver, BC V6T 1Z1 Canada

${ }^{e}$ FPInnovations, 2665 East Mall, Vancouver, BC, V6T 1 Z4 Canada

$\dagger$ Electronic supplementary information (ESI) available. CCDC 1033683 and 1033684. For ESI and crystallographic data in CIF or other electronic format see DOI: $10.1039 / \mathrm{c} 4 \mathrm{tc} 02602 \mathrm{k}$
In order to obtain materials with novel properties, e.g., in terms of stimuli responsive optical sensors, the combination of suitable host and guest materials is critical. Photonic crystals (PCs) with lattice parameters comparable to the wavelength of visible light have gained considerable attention due to their unique optical properties. ${ }^{\mathbf{1 0 , 1 1}}$ Reversible control of their optical properties by external stimuli is of crucial relevance for applications such as reflective displays or sensors. ${ }^{12,13}$ The band structure of PCs depends on both their periodicity and the refractive index contrast between their constituent dielectric materials, and can be tuned by addressing either of these parameters. For porous photonic crystals, the optical properties can be controlled by infiltration of the pores with guests of different refractive indices. With respect to this, liquid crystals (LCs) are ideal molecular guests since they show optical anisotropy due to their orientation and their refractive index can easily be addressed by external stimuli such as light or temperature..$^{\mathbf{1 0 , 1 4 - 1 7}}$ Hence, the optical properties previously locked by the host material can be altered by stimuli-responsive guests. A number of photonic crystals infiltrated with LCs have been reported, the hybrid materials showing interesting and tuneable properties. ${ }^{\mathbf{1 8 - 2 1}}$ Sato and co-workers showed control of the optical properties of an inverse opal structure loaded with LCs by photo irradiation or application of an electric field. ${ }^{\mathbf{1 0 , 1 6 , 1 7}}$ Aida and co-workers used discotic liquid crystals based on triphenylene as template for the fabrication of silica nanochannels. Due to the formation of these mesostructured silica films containing triphenylene-based charge transfer complexes in their nanochannels, they were capable of tuning the colour of the composite material with respect to the charge transfer moiety. ${ }^{22}$ 
In 2010, the MacLachlan group reported a new method to prepare high surface area mesoporous silica (CNMS), ${ }^{23}$ organosilica (CNMO) ${ }^{24}$ and carbon (CNMC) ${ }^{25}$ films with chiral nematic structures derived from cellulose nanocrystal (CNC) templating. CNMS and CNMO films selectively reflect circularly polarized light like 1D photonic crystals ${ }^{\mathbf{2 6}}$ and are strongly iridescent when their helical pitch is on the length scale of visible light. Due to the porosity, the photonic properties of these materials can be manipulated by infiltration of the mesopores with guests such as solvents or liquid crystals. CNMS films were shown to be good substrates for refractive indexbased chemical sensing. ${ }^{27}$ In another study we demonstrated reversible switching of the photonic properties of CNMO by loading its pores with a calamitic liquid crystal, 8CB ( $4^{\prime}-n$-octyl4-cyano-biphenyl). ${ }^{28,29}$ Detailed solid-state NMR spectroscopic investigations proved that the $8 \mathrm{CB}$ is aligned with the pores when it is in a mesophase.

Supramolecular liquid crystals (SMLCs) that depend on hydrogen bonding, halogen bonding, or other weak intermolecular interactions to form are an intriguing class of materials that can form many different phases. They are readily modified by switching donor/acceptor motifs. Although a number of mesoporous silica composites with LCs are known, ${ }^{17,29}$ SMLCs have not been previously investigated in mesoporous hosts. With the goal of developing temperature-sensitive photonic materials, we therefore set out to investigate SMLCs in chiral nematic mesoporous hosts.

Here we report the synthesis and characterisation of new hydrogen-bonded liquid crystals based on phloroglucinol (1,3,5-trihydroxybenzene; PG) and their injection into CNMO films (CNMO-SMLC composites) to tune the photonic properties. SMLCs chosen for the present study have two stimuliresponsive aspects, one based on their thermotropic LC properties and the other on the molecular cis-trans isomerization of either a double bond or an azo group upon irradiation with UV-light. The CNMO materials infiltrated with the hydrogenbonded liquid crystals (as shown in Scheme 1) show switchable photonic behaviour.

\section{Experimental section}

All chemicals were used as received from commercial sources without further purification. Stated yields refer to the purified and isolated products.

All spectroscopic investigations of the CNMO (composite) films were done perpendicular to the film surface. Elemental analyses were performed using an EA 1108 elemental analyser. IR spectroscopy was measured on a Nicolet 6700 FT-IR. Measurements were conducted on neat samples with an attenuated total reflectance (ATR) attachment. Mass spectra were obtained with a Kratos MS50 EI instrument. Solution NMR spectra were obtained on a Bruker AV300 or AV400dir. Solidstate ${ }^{2} \mathrm{H}$ NMR spectra were collected on a home-built NMR spectrometer based upon a $4.7 \mathrm{~T}\left({ }^{2} \mathrm{H}\right.$ resonance at $\left.30.7 \mathrm{MHz}\right)$, $89 \mathrm{~mm}$ bore magnet (Oxford Instruments, Oxford, UK). ${ }^{30}$ Spectra were collected using a quadrupolar echo pulse sequence with $3.5 \mu \mathrm{s} 90^{\circ}$ pulses, a $45 \mu \mathrm{s}$ echo delay, a spectral width of

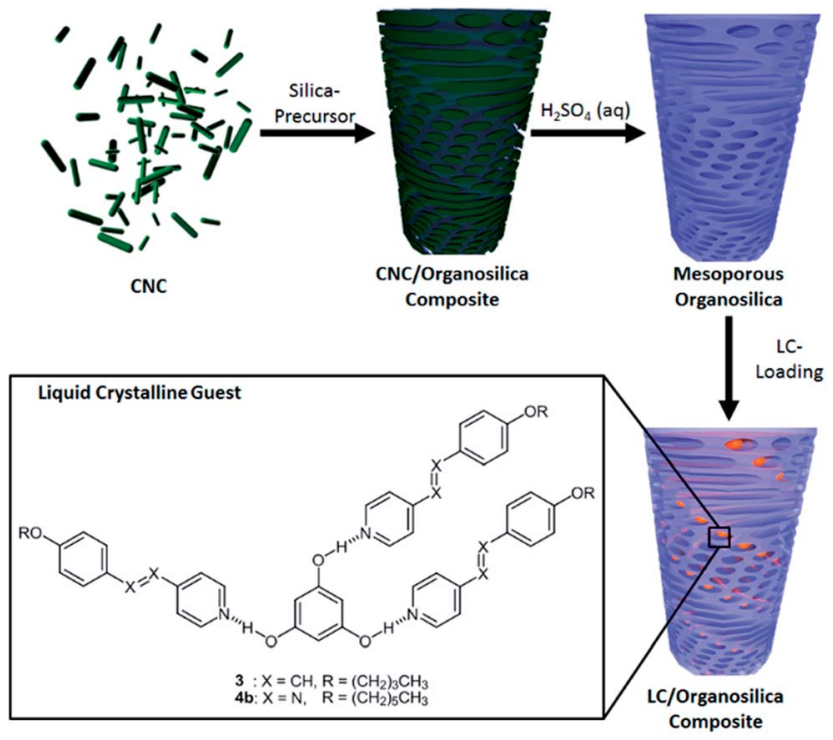

Scheme 1 Synthesis of the thermochromic CNMO-SMLC composites. CNC is used as template for chiral nematic organosilica composites. Upon removal of the CNC template under acidic conditions CNMO films are obtained, which are infiltrated by hydrogen bonded liquid crystals in order to tune the photonic properties of these novel CNMO-SMLC composites.

$1 \mathrm{MHz}$ and a $300 \mathrm{~ms}$ repetition delay. 1500 transients were averaged for each spectrum. The sample temperature was controlled using a Bruker VT-1000 temperature controller, and the sample temperature was allowed to equilibrate for at least 3 min following the temperature controller reporting stabilization at each setpoint. The temperature was initially set to $400 \mathrm{~K}$, and spectra collected in $-0.5 \mathrm{~K}$ steps. Deuteration of phloroglucinol for ${ }^{2} \mathrm{H}$ NMR spectra was done by refluxing the undeuterated compound for several days in deuterated water under an inert atmosphere. Melting points were measured with a MEL-TEMP (Capillary Melting Point Apparatus) or differential scanning calorimetry (DSC) measurements. DSC $\left(5{ }^{\circ} \mathrm{C} \mathrm{min}^{-1}\right.$ under $\mathrm{N}_{2}$ ) was conducted with PerkinElmer STA 6000. SCXRD measurements were made on a Bruker APEX DUO diffractometer with graphite monochromated Mo-K $\alpha$ radiation. UV-vis spectroscopy was carried out on a Cary 5000 UV-vis/NIR spectrophotometer equipped with an Oxford Instruments OptistatDN Cryostat (for variable temperature measurements). Polarized optical microscopy (POM) was done on an Olympus BX41 microscope equipped with an Instec hot stage. All images were taken with the sample between crossed polarizers. For the images, a Sony Cyber-shot DSC-W570 with $4.2 \times$ optical zoom was used. Experiments testing the switch-ability of the SMLCs were done by POM observation and simultaneous irradiation using a laser pointer with a wavelength of $405 \mathrm{~nm}$ and a maximal output of 5-20 $\mathrm{mW}$. Gas adsorption for BET measurements was done on a Micromeritics Accelerated Surface Area and Porosity (ASAP) 2020 system. All samples were evacuated for several hours at $120{ }^{\circ} \mathrm{C}$ prior to analysis. Measurements were related to the weight determined for the dried sample in inert dry nitrogen atmosphere. 


\section{trans-4-Hydroxy-4' -stilbazole $^{31}$}

4-Hydroxybenzaldehyde (5.04 g, $41.3 \mathrm{mmol}, 1.3 \mathrm{eq}$.) was suspended in $15 \mathrm{~mL}$ of acetic anhydride followed by the addition of $3.2 \mathrm{~mL}$ 4-picoline (32.9 mmol, 1 eq.) to form a dark brown solution. After heating the solution at reflux for $24 \mathrm{~h}$, the mixture was poured into $300 \mathrm{~mL}$ of ice water and vigorously stirred. The precipitate was filtered-off, dried and purified by recrystallization from ethanol. Afterwards, the product was heated at reflux in $70 \mathrm{~mL} \mathrm{KOH}(0.75 \mathrm{~N}$ in ethanol). Addition of acetic acid gave a yellow precipitate. After washing the filtered residue with water and ethanol, the title compound was obtained as a yellow solid. Yield: $1.62 \mathrm{~g}$ (8.20 mmol, 25\%); m.p.: $268{ }^{\circ} \mathrm{C} .{ }^{1} \mathrm{H}$ NMR (DMSO-d $\left.6,300 \mathrm{MHz}\right): \delta(\mathrm{ppm})=9.74(\mathrm{br}, \mathrm{s}, 1 \mathrm{H}$, $\mathrm{OH}), 8.48\left(\mathrm{~d}, J=6.0 \mathrm{~Hz}, 2 \mathrm{H}, \mathrm{H}_{\text {aryl }}\right), 7.43-7.53\left(\mathrm{~m}, 4 \mathrm{H}, \mathrm{H}_{\text {aryl }}\right), 7.42$ $(\mathrm{d}, J=16.5 \mathrm{~Hz}, 1 \mathrm{H}, \mathrm{CH}), 6.99(\mathrm{~d}, 16.5 \mathrm{~Hz}, 1 \mathrm{H}, \mathrm{CH}), 6.78(\mathrm{~d}, J=$ $8.7 \mathrm{~Hz}, 2 \mathrm{H}, \mathrm{H}_{\text {aryl }}$ ); ${ }^{13} \mathrm{C}$ NMR (DMSO-d 6 , $\left.75 \mathrm{MHz}\right): \delta(\mathrm{ppm})=$ 158.8, 150.5, 145.4, 133.7, 129.3, 128.1, 123.1, 121.1, 116.3; MS (EI, $50 \mathrm{eV}): m / z(\%)=197\left(100, \mathrm{C}_{13} \mathrm{H}_{11} \mathrm{NO}^{\cdot+}, \mathrm{M}^{\cdot+}\right)$; FTIR (ATR) $\nu$ $\left(\mathrm{cm}^{-1}\right)=2595,1584,1513,1422,1256,1193,1173,1005,974$, 831, 623; CHN: $\mathrm{C}_{13} \mathrm{H}_{11} \mathrm{NO}$ (197.23): C 79.16, H 5.62, N 7.10; found: C 78.76, H 5.51, N 6.73.

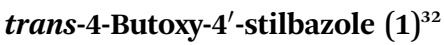

trans-4-Hydroxy-4'-stilbazole (0.51 g, $2.6 \mathrm{mmol}, 1.0$ eq.), $1.08 \mathrm{~g}$ $\mathrm{K}_{2} \mathrm{CO}_{3}$ (7.81 mmol, 3.0 eq.), and $0.56 \mathrm{~mL} n$-butylbromide (5.23 mmol, 2.0 eq.) were heated at reflux in $20 \mathrm{~mL}$ of ethanol. The reaction mixture turned from yellow to red after $\sim 20 \mathrm{~min}$. After $24 \mathrm{~h}$, the reaction mixture was cooled to room temperature and the solvent was removed under reduced pressure. The obtained residues were heated at reflux in dichloromethane for $15 \mathrm{~min}$ and undissolved solid was removed by filtration. Rotary evaporation of the organic fraction followed by column chromatography (ethyl acetate@ $\mathrm{SiO}_{2} ; R_{\mathrm{f}}=0.51$ ) afforded the pure product as a yellow solid. Yield: $0.31 \mathrm{~g}(1.24 \mathrm{mmol}, 48 \%)$; m.p.: $92{ }^{\circ} \mathrm{C} .{ }^{1} \mathrm{H}$ NMR $\left(\mathrm{CDCl}_{3}, 300 \mathrm{MHz}\right): \delta(\mathrm{ppm})=8.58(\mathrm{br}, \mathrm{s}, 2 \mathrm{H}$, $\left.\mathrm{H}_{\text {aryl }}\right), 7.52\left(\mathrm{~d}, J=8.7 \mathrm{~Hz}, 2 \mathrm{H}, \mathrm{H}_{\text {aryl }}\right), 7.47$ (br, s, 2H, $\left.\mathrm{H}_{\text {aryl }}\right), 7.35$ (d, $J=16.5 \mathrm{~Hz}, 1 \mathrm{H}, \mathrm{CH}), 6.95$ (d, $\left.J=8.7 \mathrm{~Hz}, 2 \mathrm{H}, \mathrm{H}_{\text {aryl }}\right), 6.92$ $(\mathrm{d}, J=16.5 \mathrm{~Hz}, 1 \mathrm{H}, \mathrm{CH}), 4.03\left(\mathrm{t}, J=6.5 \mathrm{~Hz}, 2 \mathrm{H}, \mathrm{OCH}_{2}\right), 1.81$ (quin., $J=7.1 \mathrm{~Hz}, 2 \mathrm{H}, \mathrm{CH}_{2}$ ), 1.53 (sext., $7.5 \mathrm{~Hz}, 2 \mathrm{H}, \mathrm{CH}_{2}$ ), 1.01 $\left(\mathrm{t}, J=7.5 \mathrm{~Hz}, 3 \mathrm{H}, \mathrm{CH}_{3}\right) ;{ }^{13} \mathrm{C} \mathrm{NMR}\left(\mathrm{CDCl}_{3}, 75 \mathrm{MHz}\right): \delta(\mathrm{ppm})=$ 160.0, 150.1 145.3, 133.1, 128.8, 128.6, 123.7, 120.8, 115.0, 68.0, 31.4, 19.4, 14.0; MS (EI, $50 \mathrm{eV}): m / z(\%)=253\left(79, \mathrm{C}_{17} \mathrm{H}_{19} \mathrm{NO}^{\cdot+}\right.$, $\left.\mathrm{M}^{\cdot+}\right), 197\left(100, \mathrm{C}_{13} \mathrm{H}_{10} \mathrm{NO}^{\cdot+}\right)$; FTIR (ATR) $\nu\left(\mathrm{cm}^{-1}\right)=2938,1588$, 1509, 1472, 1410, 1311, 1281, 1254, 1176, 1039, 1005, 971, 836, 788; $\mathrm{CHN}: \mathrm{C}_{17} \mathrm{H}_{19} \mathrm{NO}$ (253.34): C 80.60, H 7.56, N 5.53; found: $\mathrm{C}$ 80.42, H 7.43, N 5.44 .

\section{(E)-4-(Pyridin-4-yldiazenyl)phenol ${ }^{33}$}

4-Aminopyridine (6.29 g, $66.8 \mathrm{mmol}, 1.2 \mathrm{eq}$.) was dissolved in $45 \mathrm{~mL}$ of $7 \mathrm{M} \mathrm{HCl}_{(\mathrm{aq})}$ and cooled to $0{ }^{\circ} \mathrm{C}$. A solution of $5.24 \mathrm{~g}$ phenol (55.7 mmol, 1.0 eq.) and $4.23 \mathrm{~g}$ sodium nitrite (61.3 mmol, 1.1 eq.) in $20 \mathrm{~mL}$ of $10 \% \mathrm{NaOH}_{(\mathrm{aq})}$ was added dropwise to the cooled solution. Adjusting the $\mathrm{pH}$ to 6-7 by addition of base yielded an orange precipitate, which was heated in acetone to reflux. Following hot filtration, the solvent was removed by rotary evaporation to give the pure product as an orange solid (which was dried in vacuo). Yield: $3.31 \mathrm{~g}$ (1.24 mmol, 30\%); m.p.: dec. $260{ }^{\circ}$ C. ${ }^{1} \mathrm{H}$ NMR (DMSO-d 6 , $300 \mathrm{MHz}): \delta(\mathrm{ppm})=10.61(\mathrm{~s}, 1 \mathrm{H}, \mathrm{OH}), 8.77(\mathrm{~d}, J=6.1 \mathrm{~Hz}, 2 \mathrm{H}$, $\mathrm{H}_{\mathrm{ar}}$ ), $7.87\left(\mathrm{~d}, J=8.9 \mathrm{~Hz}, 2 \mathrm{H}, \mathrm{H}_{\mathrm{ar}}\right), 7.68$ (d, $J=6.1 \mathrm{~Hz}, 2 \mathrm{H}, \mathrm{H}_{\mathrm{ar}}$ ), $6.98\left(\mathrm{~d}, J=8.9 \mathrm{~Hz}, 2 \mathrm{H}, \mathrm{H}_{\mathrm{ar}}\right) ;{ }^{13} \mathrm{C} \mathrm{NMR}\left(\mathrm{DMSO}_{6}, 75 \mathrm{MHz}\right)$ : $\delta(\mathrm{ppm})=162.9,157.4,151.9,145.8,126.4,116.8$, 113.4; MS (EI, $50 \mathrm{eV}): m / z(\%)=199\left(75, \mathrm{C}_{11} \mathrm{H}_{9} \mathrm{~N}_{3} \mathrm{O}^{\cdot+}, \mathrm{M}^{\cdot+}\right), 93\left(100, \mathrm{C}_{5} \mathrm{H}_{4} \mathrm{~N}_{2}+\right.$ $\left.\mathrm{H}^{+}\right)$; FTIR (ATR) $\nu\left(\mathrm{cm}^{-1}\right)=2561,1580,1470,1404,1296,1262$, $1227,1175,1138,1010,842,828,804,732,652 ; \mathrm{CHN}^{-} \mathrm{C}_{11} \mathrm{H}_{9} \mathrm{~N}_{3} \mathrm{O}$ (199.21): C 66.32, H 4.55, N 21.09; found: C 66.40, H 4.37, N 20.79.

\section{(E)-4-((4-(Propyloxy)phenyl)diazenyl)pyridine (2a)}

(E)-4-(Pyridin-4-yldiazenyl)phenol (0.50 g, $2.51 \mathrm{mmol}, 1.0$ eq.) and $0.52 \mathrm{~g} \mathrm{~K}_{2} \mathrm{CO}_{3}$ (3.77 mmol, 1.5 eq.) were dissolved in $20 \mathrm{~mL}$ of DMF. Then $0.25 \mathrm{~mL}$ of 1-iodopropane (2.50 mmol, 1.0 eq.) were added and the mixture was stirred at $90{ }^{\circ} \mathrm{C}$ for $19 \mathrm{~h}$. The hot solution was poured into $150 \mathrm{~mL}$ deionized water yielding a red precipitate, which was filtered-off and extracted with hexane $(5 \times)$. The organic layers were combined and washed twice with $5 \% \mathrm{NaHCO}_{3(\mathrm{aq})}$ and once with saturated brine. After drying over $\mathrm{MgSO}_{4}$, the solvent was removed under reduced pressure. The obtained residue was purified by column chromatography (ethyl acetate@ $\mathrm{SiO}_{2} ; R_{\mathrm{f}}=0.69$ ). Yield: $0.17 \mathrm{~g}$ orange solid (0.60 mmol, 24\%); m.p.: $56{ }^{\circ} \mathrm{C} .{ }^{1} \mathrm{H} \mathrm{NMR}\left(\mathrm{CDCl}_{3}, 300 \mathrm{MHz}\right)$ : $\delta(\mathrm{ppm})=8.80\left(\mathrm{~d}, J=6.2 \mathrm{~Hz}, 2 \mathrm{H}, \mathrm{H}_{\mathrm{ar}}\right), 7.99(\mathrm{~d}, J=9.0 \mathrm{~Hz}, 2 \mathrm{H}$, $\mathrm{H}_{\mathrm{ar}}$ ), $7.75\left(\mathrm{~d}, J=6.2 \mathrm{~Hz}, 2 \mathrm{H}, \mathrm{H}_{\mathrm{ar}}\right), 7.06$ (d, $J=9.0 \mathrm{~Hz}, 2 \mathrm{H}, \mathrm{H}_{\mathrm{ar}}$ ), 4.05 (t, $J=7.1 \mathrm{~Hz}, 2 \mathrm{H}, \mathrm{OCH}_{2}$ ), 1.89 (sext., $J=7.1 \mathrm{~Hz}, 2 \mathrm{H}, \mathrm{CH}_{2}$ ), $1.10\left(\mathrm{t}, J=7.1 \mathrm{~Hz}, 3 \mathrm{H}, \mathrm{CH}_{3}\right) ;{ }^{13} \mathrm{C} \mathrm{NMR}\left(\mathrm{CDCl}_{3}, 300 \mathrm{MHz}\right)$ : $\delta(\mathrm{ppm})=163.3,158.0,151.0,146.8,125.8,116.5,115.1,70.1$, 22.6, 10.6; MS (EI, $50 \mathrm{eV}): m / z(\%)=241\left(64, \mathrm{C}_{14} \mathrm{H}_{15} \mathrm{~N}_{3} \mathrm{O}^{\cdot+}, \mathrm{M}^{\cdot+}\right)$, $135\left(100, \mathrm{C}_{9} \mathrm{H}_{11} \mathrm{O}^{\cdot+}\right)$; FTIR (ATR) $\nu\left(\mathrm{cm}^{-1}\right)=1578,1402,1257$, 1240, 1223, 1144, 1124, 1108, 1041, 1010, 904, 845, 792; CHN: $\mathrm{C}_{14} \mathrm{H}_{15} \mathrm{~N}_{3} \mathrm{O}$ (241.29): C 69.69, H 6.27, N 17.41; found: C 69.68, H 6.13, N 17.29.

\section{(E)-4-((4-(Hexyloxy)phenyl)diazenyl)pyridine (2b) $)^{34}$}

(E)-4-(Pyridin-4-yldiazenyl)phenol (1.00 g, $5.05 \mathrm{mmol}, 1.0$ eq.) and potassium carbonate $(1.05 \mathrm{~g}, 7.58 \mathrm{mmol}, 1.5 \mathrm{eq}$.$) were$ dissolved in $20 \mathrm{~mL}$ dimethylformamide followed by the addition of $0.75 \mathrm{~mL}$ of 1-iodohexane $(5.05 \mathrm{mmol}, 1.0$ eq.). The solution was heated to $90{ }^{\circ} \mathrm{C}$ for $19 \mathrm{~h}$ and then poured hot into $150 \mathrm{~mL}$ deionized water, leading to a red precipitate. The solid was isolated by filtration then triturated with hexane $(5 \times)$. The organic fractions were combined and washed twice with sodium bicarbonate $(5 \%)$ and once with saturated brine. After drying the organic phase over magnesium sulfate, the mixture was filtered and the solvent was removed under reduced pressure. Purification by column chromatography (ethyl acetate@SiO $\mathrm{S}_{2} ; R_{\mathrm{f}}$ $=0.67)$ yielded the desired product. Yield: $0.95 \mathrm{~g}$ orange solid (3.35 mmol, 66\%); m.p.: $56{ }^{\circ} \mathrm{C} .{ }^{1} \mathrm{H} \mathrm{NMR}\left(\mathrm{CDCl}_{3}, 300 \mathrm{MHz}\right)$ : $\delta(\mathrm{ppm})=8.81\left(\mathrm{~d}, J=5.8 \mathrm{~Hz}, 2 \mathrm{H}, \mathrm{H}_{\mathrm{ar}}\right), 8.00(\mathrm{~d}, J=8.9 \mathrm{~Hz}, 2 \mathrm{H}$, $\left.\mathrm{H}_{\mathrm{ar}}\right), 7.75\left(\mathrm{~d}, J=5.8 \mathrm{~Hz}, 2 \mathrm{H}, \mathrm{H}_{\mathrm{ar}}\right), 7.05\left(\mathrm{~d}, J=8.9 \mathrm{~Hz}, 2 \mathrm{H}, \mathrm{H}_{\mathrm{ar}}\right.$ ), 4.09 (t, $J=7.1 \mathrm{~Hz}, 2 \mathrm{H}, \mathrm{OCH}_{2}$ ), 1.86 (quin., $J=7.1 \mathrm{~Hz}, 2 \mathrm{H}, \mathrm{CH}_{2}$ ), 1.45-1.59 (m, 2H, $\mathrm{CH}_{2}$ ), 1.32-1.44 (m, 4H, $\left.\mathrm{CH}_{2}\right), 0.95$ (t, $J=7.1$ 
$\left.\mathrm{Hz}, 2 \mathrm{H}, \mathrm{CH}_{3}\right) ;{ }^{13} \mathrm{C} \mathrm{NMR}\left(\mathrm{CDCl}_{3}, 75 \mathrm{MHz}\right): \delta(\mathrm{ppm})=163.3,158.0$, 150.7, 146.7, 125.9, 116.6, 115.1, 68.7, 31.7, 29.2, 25.8, 22.7, 14.2; MS (EI, $50 \mathrm{eV}): m / z(\%)=283\left(71, \mathrm{C}_{17} \mathrm{H}_{21} \mathrm{~N}_{3} \mathrm{O}^{\cdot+}, \mathbf{M}^{\cdot+}\right), 177$ $\left(100, \mathrm{C}_{12} \mathrm{H}_{17} \mathrm{O}^{\circ+}\right)$; FTIR (ATR) $\nu\left(\mathrm{cm}^{-1}\right)=2936,2857,1602,1583$, 1503, 1469, 1410, 1254, 1137, 1028, 837, 794, 728, 635; CHN: $\mathrm{C}_{17} \mathrm{H}_{21} \mathrm{~N}_{3} \mathrm{O}$ (283.87): C 72.06, H 7.47, N 14.83; found: C 71.89, $\mathrm{H}$ 7.53, N 14.63.

\section{[trans-4-Butoxy-4' ${ }^{\prime}$-stilbazole/PG $]_{3: 1}(3)^{35}$}

Phloroglucinol (5-10 mg) was mixed with trans-4-butoxy-4'-stilbazole (1) in a $1: 3$ ratio and dissolved in acetone. Slow evaporation of acetone in a dry atmosphere overnight leads to the hydrogen-bonded assembly. Quantitative yield; m.p.: $126{ }^{\circ} \mathrm{C}$ (dec.). ${ }^{1} \mathrm{H}$ NMR (acetone- $\left.\mathrm{d}_{6}, 600 \mathrm{MHz}\right): \delta(\mathrm{ppm})=8.50(\mathrm{~d}, J=6.0$ $\mathrm{Hz}, 6 \mathrm{H}, \mathrm{H}_{\text {aryl }}$ ), 8.13 (br, s, 3H, OH), 7.57 (d, J=9.0 Hz, 6H, $\mathrm{H}_{\text {aryl }}$ ), 7.47 (d, $J=6.0 \mathrm{~Hz}, 6 \mathrm{H}, \mathrm{H}_{\text {aryl }}$ ), 7.46 (d, $\left.J=16.8 \mathrm{~Hz}, 1 \mathrm{H}, \mathrm{CH}\right), 7.06$ (d, $J=16.8 \mathrm{~Hz}, 1 \mathrm{H}, \mathrm{CH}), 6.95$ (d, $\left.J=9.0 \mathrm{~Hz}, 6 \mathrm{H}, \mathrm{H}_{\text {aryl }}\right), 5.88$ (s, 3H, $\mathrm{H}_{\text {aryl }}$ ), 4.02 (t, $J=7.5 \mathrm{~Hz}, 6 \mathrm{H}, \mathrm{CH}_{2}$ ), 1.75 (quint., $J=7.5$ $\mathrm{Hz}, 6 \mathrm{H}, \mathrm{CH}_{2}$ ), 1.49 (sext., $J=7.5 \mathrm{~Hz}, 6 \mathrm{H}, \mathrm{CH}_{2}$ ), 0.96 (t, $J=7.5 \mathrm{~Hz}$, $\left.9 \mathrm{H}, \mathrm{CH}_{3}\right) ;{ }^{13} \mathrm{C}$ NMR (acetone- $\left.\mathrm{d}_{6}, 75 \mathrm{MHz}\right): \delta(\mathrm{ppm})=159.4$, 158.8, 149.6, 144.6, 132.3, 128.5, 128.0, 123.0, 120.1, 114.3, 94.0, 67.0, 28.9, 18.5, 12.8; FTIR (ATR) $\nu\left(\mathrm{cm}^{-1}\right)=3030,2957,2933$, 2871, 2635, 1593, 1515, 1247, 1177, 1158, 1148, 998, 967, 827, 805, 783; CHN: $\mathrm{C}_{57} \mathrm{H}_{63} \mathrm{~N}_{3} \mathrm{O}_{6}$ (886.13): C 77.26, $\mathrm{H}$ 7.17, N 4.74; found: C 76.85, H 7.43, N 4.61; CCDC: 1033683.

\section{[(E)-4-((4-(propyloxy)phenyl)diazenyl)pyridine/PG $]_{3: 1}$ (4a)}

Phloroglucinol (5-10 mg) was mixed with (E)-4-((4-(propyloxy)phenyl)diazenyl)pyridine (2a) in a $1: 3$ ratio and dissolved in acetone. Slow evaporation of acetone in a dry atmosphere overnight leads to the hydrogen-bonded assembly. Quantitative yield; m.p.: 133-135 ${ }^{\circ} \mathrm{C}$, under decomposition. ${ }^{1} \mathrm{H}$ NMR (acetone- $\left.\mathrm{d}_{6}, 600 \mathrm{MHz}\right): \delta(\mathrm{ppm})=8.78\left(\mathrm{~d}, J=6.2 \mathrm{~Hz}, 6 \mathrm{H}, \mathrm{H}_{\text {aryl }}\right)$, $8.13(\mathrm{~s}, 3 \mathrm{H}, \mathrm{OH}), 7.97$ (d, $\left.J=9.0 \mathrm{~Hz}, 6 \mathrm{H}, \mathrm{H}_{\text {aryl }}\right), 7.70(\mathrm{~d}, J=6.2$ $\mathrm{Hz}, 6 \mathrm{H}, \mathrm{H}_{\text {aryl }}$ ), 7.70 (d, $\left.J=6.2 \mathrm{~Hz}, 6 \mathrm{H}, \mathrm{H}_{\text {aryl }}\right), 7.13$ (d, $J=9.0 \mathrm{~Hz}$, $\left.6 \mathrm{H}, \mathrm{H}_{\text {aryl }}\right), 5.89$ (s, 3H, $\left.\mathrm{H}_{\text {aryl }}\right), 4.08$ (t, $\left.J=6.5 \mathrm{~Hz}, 6 \mathrm{H}, \mathrm{CH}_{2}\right), 1.82$ (sext., $J=6.5 \mathrm{~Hz}, 6 \mathrm{H}, \mathrm{CH}_{2}$ ), $1.03\left(\mathrm{t}, J=6.5 \mathrm{~Hz}, 9 \mathrm{H}, \mathrm{CH}_{3}\right) ;{ }^{13} \mathrm{C}$ NMR (acetone- $\left.\mathrm{d}_{6}, 75 \mathrm{MHz}\right): \delta(\mathrm{ppm})=162.7,158.8,156.8,150.9$, 146.1, 125.0, 115.4, 114.6, 94.0, 69.3, 21.8, 9.3; FT-IR (ATR) $\nu\left(\mathrm{cm}^{-1}\right)=3046,2967,2923,2880,2615,1592,1496,1404,1257$, 1144, 1002, 969, 835, 634; CHN: $\mathrm{C}_{48} \mathrm{H}_{51} \mathrm{~N}_{9} \mathrm{O}_{6}$ (849.98): C 67.83, H 6.05, N 14.83; found: C 67.96, H 5.96, N 14.74; CCDC: 1033684.

\section{[(E)-4-((4-(Hexyloxy)phenyl)diazenyl)pyridine/PG $]_{3: 1}$ (4b)}

Phloroglucinol (5-10 mg) was mixed with (E)-4-((4-(hexyloxy)phenyl)diazenyl)pyridine (2b) in a $1: 3$ ratio and dissolved in acetone. Slow evaporation of acetone in a dry atmosphere overnight leads to the hydrogen-bonded assembly. Yield: quantitative yield; m.p.: dec. $100{ }^{\circ} \mathrm{C} .{ }^{1} \mathrm{H}$ NMR (acetone- $\mathrm{d}_{6}$, $600 \mathrm{MHz}): \delta(\mathrm{ppm})=8.81\left(\mathrm{~d}, J=5.8 \mathrm{~Hz}, 2 \mathrm{H}, \mathrm{H}_{\mathrm{ar}}\right), 8.00(\mathrm{~d}, J=8.9$ $\left.\mathrm{Hz}, 2 \mathrm{H}, \mathrm{H}_{\mathrm{ar}}\right), 7.75\left(\mathrm{~d}, J=5.8 \mathrm{~Hz}, 2 \mathrm{H}, \mathrm{H}_{\mathrm{ar}}\right), 7.05(\mathrm{~d}, J=8.9 \mathrm{~Hz}, 2 \mathrm{H}$, $\mathrm{H}_{\mathrm{ar}}$ ), 4.09 (t, $J=7.1 \mathrm{~Hz}, 2 \mathrm{H}, \mathrm{OCH}_{2}$ ), 1.86 (quin., $J=7.1 \mathrm{~Hz}, 2 \mathrm{H}$, $\left.\mathrm{CH}_{2}\right)$, 1.45-1.59 (m, 2H, $\left.\mathrm{CH}_{2}\right), 1.32-1.44\left(\mathrm{~m}, 4 \mathrm{H}, \mathrm{CH}_{2}\right), 0.95$ $\left(\mathrm{t}, J=7.1 \mathrm{~Hz}, 2 \mathrm{H}, \mathrm{CH}_{3}\right) ;{ }^{13} \mathrm{C}$ NMR (acetone- $\left.\mathrm{d}_{6}, 75 \mathrm{MHz}\right): \delta(\mathrm{ppm})$ $=162.7$, 158.8, 156.8, 150.9, 146.1, 125.0, 115.0, 114.6, 94.0, 67.9, 30.9, 28.2, 25.0, 21.9, 13.0; FT-IR (ATR) $\nu\left(\mathrm{cm}^{-1}\right)=3050$,
2948, 2932, 2864, 2635, 1592, 1498, 1405, 1251, 1141, 1000, 839, 792; $\mathrm{CHN}: \mathrm{C}_{57} \mathrm{H}_{69} \mathrm{~N}_{9} \mathrm{O}_{6}$ (976.21): C 70.13, H 7.12, N 12.91; found: C 70.40, H 7.03, N 12.93 .

\section{Results and discussion}

\section{Synthesis and characterisation of the hydrogen bonded assemblies}

trans-4-Butoxy-4'-stilbazole derivative 1 (ref. 31, 32, 36 and 37) and trans-4-alkoxy-4'-azobenzene derivatives $\mathbf{2 a}$ and $\mathbf{2 b}$ (ref. 33 and 34) were synthesized according to known procedures and fully characterized by ${ }^{1} \mathrm{H}$ NMR, ${ }^{13} \mathrm{C}$ NMR and IR spectroscopy, mass spectrometry and elemental analysis (EA). In order to obtain the hydrogen-bonded assemblies, phloroglucinol was mixed with either $\mathbf{1}, \mathbf{2 a}$ or $\mathbf{2 b}$ in a $1: 3$ ratio in anhydrous acetone, giving $\mathbf{3}, \mathbf{4 a}$ and $\mathbf{4 b}$, respectively, upon slow evaporation of the solvent in a moisture-free environment (Scheme 2). ${ }^{35}$

To the best of our knowledge, no liquid crystallinity has been reported for pristine compounds $\mathbf{1}$ and $\mathbf{2 b}$. Furthermore, we could not observe any mesophase in these compounds by polarized optical microscopy or differential scanning calorimetry (DSC) (Fig. S1†). However, as soon as phloroglucinol was introduced to hydrogen bond to the pyridine rings, mesomorphism was observed for compounds $\mathbf{3}$ and $\mathbf{4 b}$ upon cooling from the isotropic state (as seen by the schlieren texture in Fig. 1). Hence, the LC properties of $\mathbf{3}$ and $\mathbf{4 b}$ arise directly from the hydrogen-bonded assemblies formed between the phloroglucinol core unit and the peripheral pyridine moieties. The formation of the hydrogen-bonded assemblies 3, $\mathbf{4 a}$ and $\mathbf{4 b}$ was proven by NMR spectroscopy, EA and IR spectroscopy. In addition, IR spectroscopy verifies the hydrogen bonding as the $\mathrm{OH}$ absorption bands shifted to lower energy. Pristine phloroglucinol has a broad $\mathrm{OH}$ vibration mode at $3224 \mathrm{~cm}^{-1}$ while the final assemblies 3, $\mathbf{4 a}$ and $\mathbf{4 b}$ exhibit a broad signal with interstratified sharp signals between $2630 \mathrm{~cm}^{-1}$ and $3050 \mathrm{~cm}^{-1}$ (Fig. S2†).

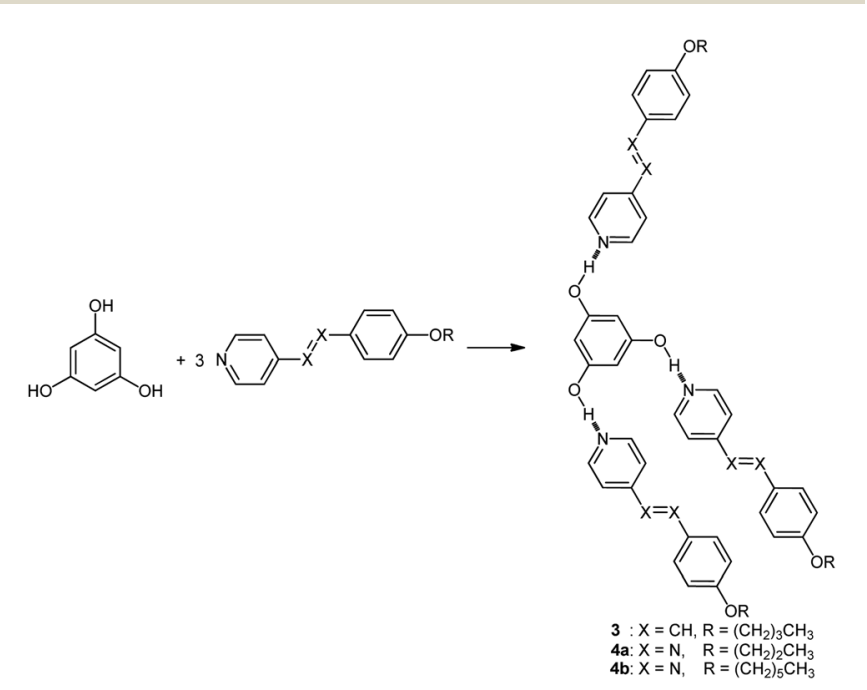

Scheme 2 Synthesis of the hydrogen-bonded assemblies 3, 4a and $4 b$. 


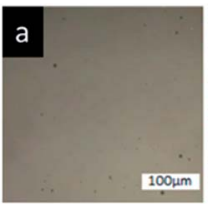

$\sim 135^{\circ} \mathrm{C}$ (ISO)

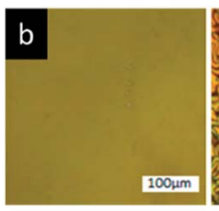

$\sim 90{ }^{\circ} \mathrm{C}$ (ISO)

$\sim 85^{\circ} \mathrm{C}\left(\mathrm{N}_{\text {col }}\right)$

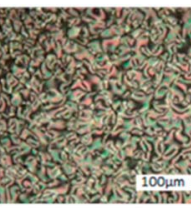

$\sim 115^{\circ} \mathrm{C}\left(\mathrm{N}_{\mathrm{col}}\right)$
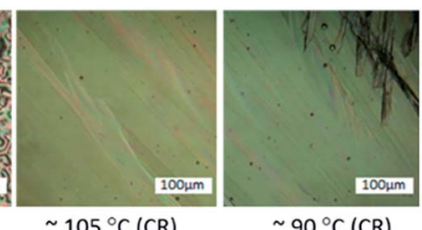

$\sim 90{ }^{\circ} \mathrm{C}$ (CR)

$\sim 105^{\circ} \mathrm{C}(\mathrm{CR})$

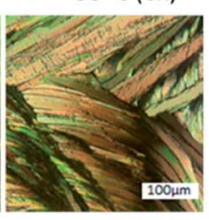

$\sim 25^{\circ} \mathrm{C}(\mathrm{CR})$
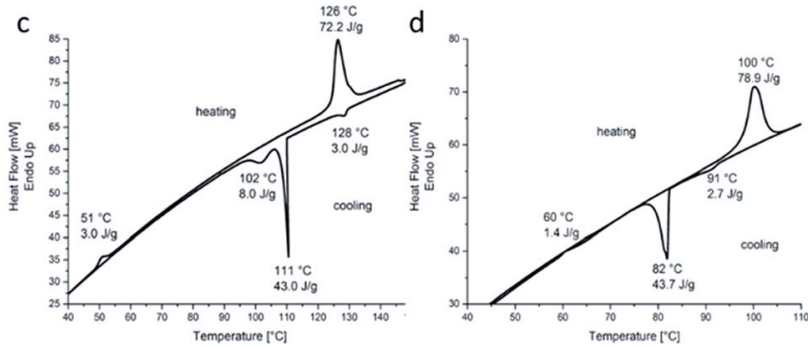

Fig. 1 Polarized optical micrographs of 3 (a) and 4b (b) upon cooling from the isotropic melt reveal the liquid crystalline behaviour of the compounds. (c), (d) DSC profiles of 3 and $4 \mathrm{~b}$ for both heating and cooling cycles.

Lee et al. already reported the liquid crystalline properties of $3,{ }^{35,38} 124{ }^{\circ} \mathrm{C}\left(\right.$ ISO- $\left.\mathrm{N}_{\mathrm{col}}\right)$ and $91{ }^{\circ} \mathrm{C}\left(\mathrm{N}_{\text {col }} \mathrm{CR}\right)$; however, we found slightly different transition temperatures for the phase transitions at $128{ }^{\circ} \mathrm{C}$ (ISO-LC) and $102{ }^{\circ} \mathrm{C}$ (LC-CR) by DSC. These differences may be attributed to calibration issues of our setup (compare to solid-state NMR studies below).

By synthesizing the trans-4-alkoxy-4'-azobenzene assemblies 4a and $\mathbf{4 b}$ we were able to expand the range of accessible hydrogen-bonded liquid crystals and to introduce a fast and reversible photo-switch. Compound 4a, which was prepared only as a model, does not exhibit liquid crystalline properties as proven by variable temperature POM. Similar to 3 , compound 4b exhibits a monotropic LC phase transition upon cooling from the melted state. The transitions occur at $91{ }^{\circ} \mathrm{C}$ (ISO-LC) and $82{ }^{\circ} \mathrm{C}$ (LC-CR).

Stilbazole $\mathbf{3}$ and azobenzene $\mathbf{4 b}$ undergo cis-trans isomerization upon UV irradiation, which offers external control of the alignment of the assemblies. The phase transitions of the liquid-crystalline assemblies from the LC phase to the isotropic mesophases upon UV light irradiation with a laser pointer were investigated by POM (Fig. 2).

The LC phase disappears rapidly for both assemblies 3 and 4b upon irradiation. Thus, photoisomerization from the transstate exhibiting liquid crystalline properties to the isomeric cis-state leads to structural changes that disrupt the ordered alignment. The nematic phase reappears within a few minutes (SMLC 3) after the light source is removed, indicating a
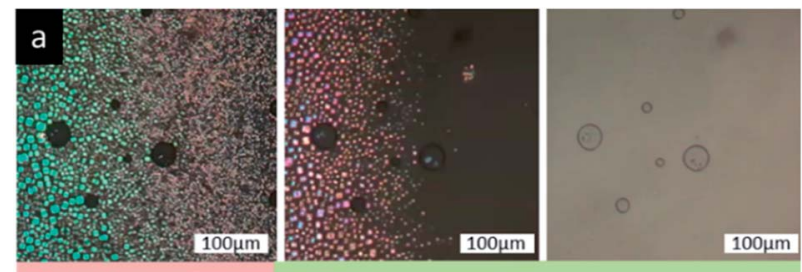

Laser off
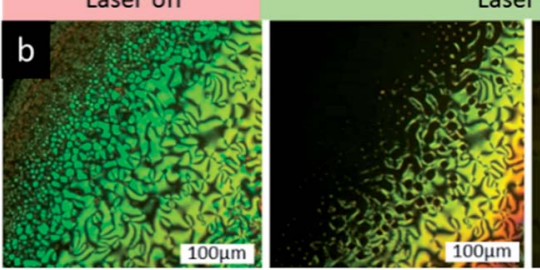

ser on

Fig. 2 Photo-initiated phase transition $\mathrm{N} \rightarrow$ ISO of SMLC 3 (a) and 4b (b) upon irradiation with a laser pointer (purple, $\lambda=405 \mathrm{~nm}$ ).

reversible process. In the case of SMLC $\mathbf{4 b}$, the nematic phase begins to re-establish within seconds.

To get deeper insight into the structural features of the mesogens, single crystals of $\mathbf{3}$ and $\mathbf{4 a}$ suitable for X-ray diffraction analysis were obtained from acetone. Fig. 3 shows the space-filling diagram for $\mathbf{3}$ and $\mathbf{4 a}$, illustrating their solid-state packing.

Compound 3 crystallizes from acetone as yellow tablets in the space group $P \overline{1}$ with two crystallographically independent molecules in the asymmetric unit. In one formula unit, one of the pyridine ligands is disordered in two orientations. In the case of the ordered formula unit of 3, the distance of the hydrogen bridges (ipso-C- $\mathrm{N}_{\text {pyr }} \cdots \mathrm{OH}$ ) is between $2.72-2.77 \AA$ for all three ligands. Two of them are nearly coplanar with the phloroglucinol ring with tilt angles of $20.9^{\circ}$ and $23.9^{\circ}$, respectively, while the third ligand is tilted $81.2^{\circ}$ out of plane. The angles are determined between the planes of the pyridine moieties with the plane of the phloroglucinol unit. The solidstate packing of 3 shows the edge-to-face arrangement of the

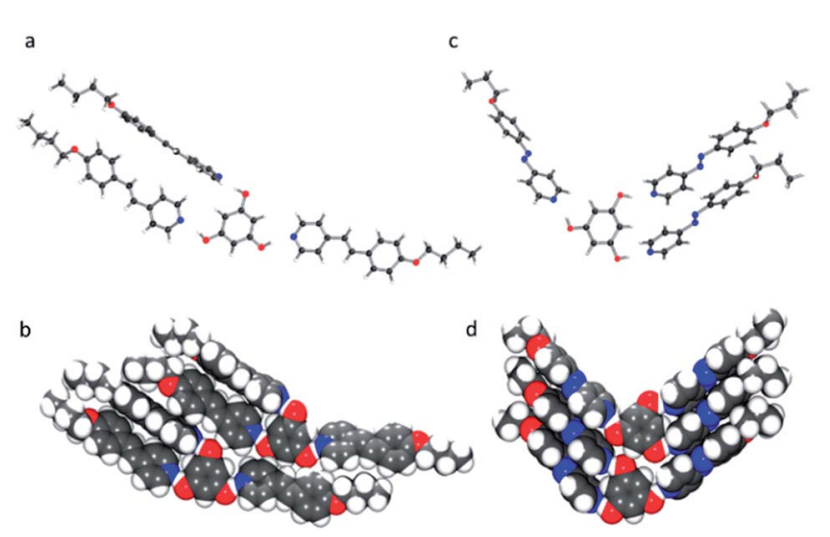

Fig. 3 (a) Molecular structure of 3, (b) plot showing the intermolecular interactions in 3 in the solid state, (c) molecular structure of $4 a$, (d) plot showing the intermolecular interactions in $4 a$ in the solid state. Disordered molecules are omitted for clarity (C: grey, $\mathrm{H}$ : white, $\mathrm{N}$ : blue and O: red). 
arenes within the assembly ( $\mathrm{CH} \cdots$ centroid $=2.85-2.96 \AA)$ as well as between adjacent assemblies $(\mathrm{CH} \cdots$ centroid $=2.86 \AA$ ).

Compound $\mathbf{4 a}$ crystallizes as red prisms in the space group $P 2_{1} / n$. Hydrogen-bonded assembly 4 a consists of only one molecule in the asymmetric unit without any disorder. The hydrogen bridges in $\mathbf{4 a}$ (ipso-C- $\mathrm{N}_{\mathrm{pyr}} \cdots \mathrm{OH}$ ) are between 2.75-2.81 $\AA$, slightly longer than those in 3. Furthermore, the three ligands of $4 \mathbf{a}$ are all oriented out of plane of the phloroglucinol ring, exhibiting tilt angles of $59.4^{\circ}, 65.0^{\circ}$ and $72.0^{\circ}$ between the pyridine ring planes and the phloroglucinol ring plane. Two out of the three hydrogen-bonded side chains are almost coplanar with only $7.2^{\circ}$ between the two planes spanned by the pyridine groups. In contrast to compound $\mathbf{3}$, the solidstate intermolecular packing of $4 a$ shows shifted face-to-face interaction of the arenes of the diazobenzene units with distances of 3.75-3.83 $\AA^{39}$

In both crystal structures ( $\mathbf{3}$ and $\mathbf{4 a}$ ) all non-hydrogen atoms were refined anisotropically and all $\mathrm{OH}$ hydrogen atoms were located in difference maps and refined isotropically, showing that no deprotonation by the pyridine unit has occurred. Both structures for $\mathbf{3}$ and $\mathbf{4 a}$ clearly depict the hydrogen bonding between the nitrogen of each pyridine moiety with a hydroxy group of phloroglucinol as shown in Fig. 3. It is interesting to note that in the crystal structures for both assemblies $\mathbf{3}$ and $\mathbf{4 a}$, the alignment of the peripheral units towards the phloroglucinol resemble a more rodlike structure rather than a discotic one.

However, the solid-state structures of $\mathbf{3}$ or $\mathbf{4 a}$ do not represent the structural features of the mesophases of $\mathbf{3}$ and $\mathbf{4 b}$. As reported by Lee et al., ${ }^{35}$ [trans-4-butoxy- $4^{\prime}$-stilbazole/PG $]_{3: 1}$ 3 upon cooling from the isotropic phase shows a phase sequence, $124.1{ }^{\circ} \mathrm{C}\left(\mathrm{ISO}-\mathrm{N}_{\mathrm{col}}\right)$ and $91.0{ }^{\circ} \mathrm{C}\left(\mathrm{N}_{\mathrm{col}}{ }^{-\mathrm{CR}}\right)$, where the monotropic columnar nematic $\left(\mathrm{N}_{\mathrm{col}}\right)$ phase has been identified by POM and X-ray diffraction studies. These authors showed that the three-armed systems formed by simple hydrogen bonding can form discotic columnar phases $\left(\mathrm{N}_{\text {col }}\right.$ or hexagonal $D_{\mathrm{h}}$ for longer alkyl chains) through self-assembly. However the ordering and alignment of $[\text { trans-4-butoxy-4'-stilbazole/PG }]_{3: 1}$ 3 molecules in the $\mathrm{N}_{\text {col }}$ phase remain unexplored. In order to get a deeper understanding of the alignment and stacking of these mesogens in the $\mathrm{N}_{\text {col }}$ phase, the PG ring of 3 was deuterated to allow a study by means of solid-state deuterium NMR spectroscopy. Representative solid-state ${ }^{2} \mathrm{H}$ NMR spectra as a function of temperature during cooling are shown in Fig. 4a.

The spectra in the $\mathrm{N}_{\text {col }}$ phase consist of two sharp quadrupolar doublets, having slightly asymmetric line shapes. The asymmetry reflects a planar distribution of directors that lie in (and slightly out of) the plane perpendicular to the magnetic field (see Fig. 5a). Such a planar behavior is typical for discotic systems. ${ }^{\mathbf{4 0 4 1}}$ Apart from the biphasic region close to $T_{\mathrm{C}}$ $\left(\sim 127^{\circ} \mathrm{C}\right.$ ), these spectra, collected by cooling 3 from the I phase, show two distinct quadrupolar splittings: one $\left(\Delta \nu_{2}\right)$ is for the CD deuterons on the PG ring, while the larger one $\left(\Delta \nu_{1}\right)$, showing a broader line width, is for the hydroxyl deuterons. At $113{ }^{\circ} \mathrm{C}$, the sample has entered into a crystalline phase, where the doublets have disappeared and are replaced by a broad powder pattern together with a relatively sharp DNMR line in the center (not
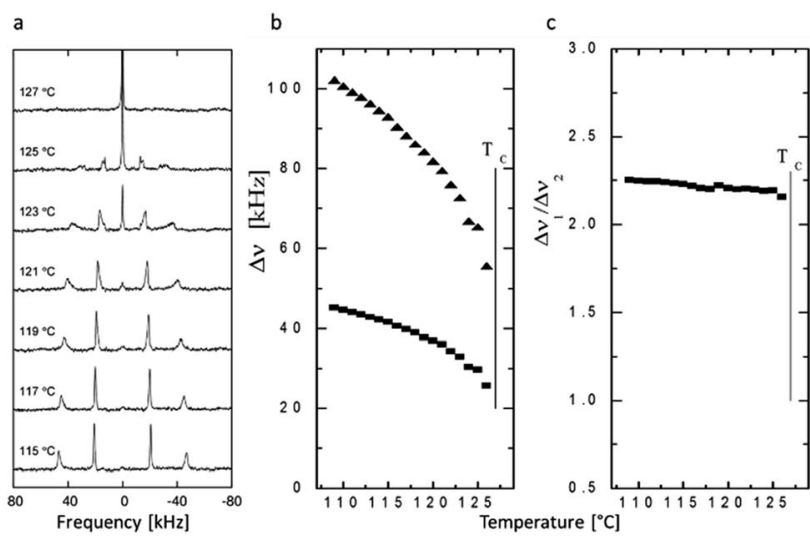

Fig. 4 Representative ${ }^{2} \mathrm{H}$ quadrupolar-echo NMR spectra of 3 as a function of temperature when 3 is cooled from the isotropic melt (a). Plots of quadrupolar splittings (b) and their ratio (c) as a function of temperature in the $\mathrm{N}_{\text {col }}$ phase of 3.

shown). Fig. $4 \mathrm{~b}$ shows a plot of the quadrupolar splittings as a function of temperature, while the ratio between these splittings, which is nearly independent of temperature, is shown in Fig. 4c.

Based on these observations, one can postulate a model for how these LC molecules are stacked in the $\mathrm{N}_{\text {col }}$ phase (Fig. 5a).

Each $[\text { trans-4-butoxy-4'-stilbazole/PG }]_{3: 1} 3$ component in the nematic phase adopts a 'bowl' shape ${ }^{42,43}$ with the three pyridylcontaining groups hydrogen bonding to the central PG ring (see Fig. 5a). This conformation differs from the above single crystal X-ray structure but allows easy self-assembly of molecules into columns whose symmetry axes are preferentially ordered along a local director. For deuterons (CD or OD) on the PG ring, the quadrupolar splitting of $i^{\text {th }}$ deuteron(s) after assuming the order of PG is axially symmetric (i.e. $S_{x x}-S_{y y}=0$ ) is given by ${ }^{44}$

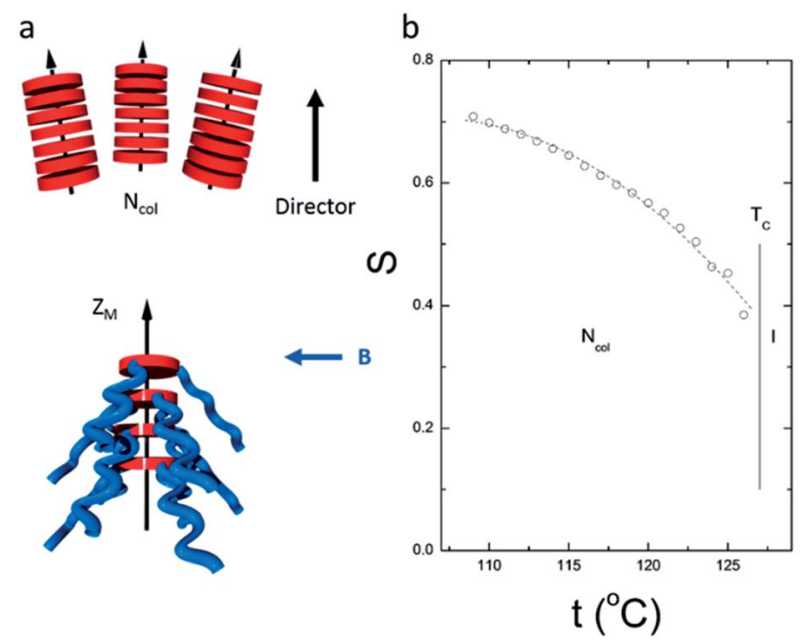

Fig. 5 Schematic representation of the alignment of the liquid crystalline assembly within the magnetic field (a) as well as the nematic order parameter $S$ versus temperature in the $N_{\text {col }}$ phase of 3 (b). Dashed line is drawn to aid the eyes. 


$$
\Delta \nu_{i}=-(3 / 8) q_{b b}{ }^{i}\left\{S_{z z}\left[\left(3 l_{z b}{ }^{2}-1\right)+\eta\left(l_{z a}{ }^{2}-l_{z c}{ }^{2}\right)\right]\right\}
$$

where $l_{z j}$ is the cosine of angle between the $j$ axis of the principal axis $(a, b, c)$ system of the quadrupole interaction and the molecular symmetry $z$ axis (normal to the ring plane) of the PG ring, $\eta$ is its asymmetry parameter and $q_{b b}$ is the quadrupolar coupling constant ( $b$ axis is along CD or OD bond). The above equation has taken into account the fact that the director is perpendicular to the external $B$ field and the assumption made is generally accepted as a good approximation for discotic systems. Hence, for deuterons of the hydroxyl group $(\angle \mathrm{COD}=$ $111.3^{\circ}$, a value for phenol-acetone $\left.e^{45}\right)$, one has $l_{z b}=\cos 21.3^{\circ}$, $l_{z a}=\sin 21.3^{\circ}$ and $l_{z c}=0$, which gives

$$
\Delta \nu_{1}=-(3 / 8) q^{\mathrm{OD}} S_{z z}\left[\left(3 \cos ^{2} 21.3^{\circ}-1\right)+\eta \sin ^{2} 21.3^{\circ}\right] .
$$
$\mathrm{be}^{40}$

Similarly one finds the splitting $\Delta \nu_{2}$ for the CD deuteron to

$$
\Delta \nu_{2}=(3 / 8) q^{\mathrm{CD}} S_{z z}(1-\eta) .
$$

For the latter deuteron(s), typical values for $q^{\mathrm{CD}}=180 \mathrm{kHz}$ and $\eta=0.045$ (ref. 41) are taken here, while for the former one, a typical $\eta=0.15$ is used. ${ }^{46}$ Since Fig. $5 \mathrm{~b}$ has a $\Delta \nu_{1} / \Delta \nu_{2}=2.23$, this allows us to determine $q^{\mathrm{OD}}$, yielding a value of $236 \mathrm{kHz}$. This derived quadrupolar coupling constant is in excellent agreement with the value of $232 \mathrm{kHz}$ reported for 2,4-dichlorophenol. ${ }^{46}$ The above results lend support to the fact that the three-armed system could indeed adopt a 'bowl-like' conformation. In particular the PG central unit is rigid and the overall molecular shape is consistent with stacking into columns arranged in a nematic fashion. The nematic behaviour is further borne out by the obtained nematic order parameter $S_{z z}=S$ shown in Fig. 5b using either eqn (2) or (3).

The $S$ value increases from a value of $c a .0 .38$ at $T_{\mathrm{C}}$ with decreasing temperature reaching a value of 0.7 at the low end of the nematic range. This behaviour is consistent with that seen for classical LCs and in particular values obtained by Monte Carlo simulation of a bowl-shaped LC. ${ }^{47}$

\section{Preparation and characterisation of the CNMO-SMLC composite films}

Our aim was to infiltrate CNMO films in order to obtain novel hybrid materials with stimulus-controlled photonic properties (thermoresponsive and photoswitchable). Therefore, ethylenebridged chiral nematic mesoporous organosilica films were synthesized according to a previously reported procedure. ${ }^{24} \mathrm{~A}$ mixture of 1,2-bis(trimethoxysilyl)ethane (BTMSE) and CNCs in water was allowed to dry under ambient conditions to yield freestanding composite films. Upon removal of the CNC template using acid hydrolysis in $\mathrm{H}_{2} \mathrm{SO}_{4(\mathrm{aq})}$ followed by oxidative treatment with $\mathrm{H}_{2} \mathrm{O}_{2} / \mathrm{H}_{2} \mathrm{SO}_{4(\mathrm{aq})}$ to remove insoluble cellulosic by-products, yellow-orange iridescent films were obtained. The wavelength $\lambda_{\max }$ corresponding to the position of peak reflection by a chiral nematic material is given by

$$
\lambda_{\max }=n_{\mathrm{avg}} P \sin (\theta)
$$

where $n_{\text {avg }}$ is the average refractive index of the material, $P$ the length of the helical pitch and $\theta$ the angle of incident light relative to plane perpendicular to $P$.

The obtained CNMO films were characterized by SEM images, UV-vis and CD spectroscopy as well as BET and TGA measurements. The chiral nematic organisation was retained in the pore structure as evidenced by SEM images and a strong reflection peak at $602 \mathrm{~nm}$ observed by both UV-vis and CD spectroscopy (Fig. S3 $\dagger$ ). BET analysis of $\mathrm{N}_{2}$ adsorption data indicate that the CNMO films have a surface area of $417 \mathrm{~m}^{2} \mathrm{~g}^{-1}$, reflecting the high porosity of the material. Also, an average pore diameter of $6.4 \mathrm{~nm}$ was determined. These results show that the CNMO films are highly suitable to act as host material for small molecular compounds. TGA measurements of the CNMO prove that the films are stable to $380{ }^{\circ} \mathrm{C}$ (Fig. S4†).

SMLCs were infiltrated into the mesopores of the CNMO films in the melt. Prior to the infiltration, neutralization of the CNMO films by base treatment with aqueous ammonium hydroxide solution (3\%) was necessary to prevent disturbance of the hydrogen-bridged assembly due to interactions with the silica based host material/formation of pyridinium salts. Any excess of SMLCs remaining on the surface of the films was wiped off several times prior to analytical investigation.

TGA data indicated that the CNMO films contained up to $27 \mathrm{wt} \%$ of compound 3 (Fig. S5 $\dagger$ ). The infiltration of the pores leads to a red shift of the reflection signal from $602 \mathrm{~nm}$ to $727 \mathrm{~nm}$, consistent with replacing air in the channels with the higher refractive index guest, 3. Investigation of the guest's cis-trans isomerization by irradiation with a laser pointer, as done for the bulk materials $\mathbf{3}$ and $\mathbf{4 b}$, was not successful. Furthermore, the CNMO-SMLC $\mathbf{4 b}$ composite films incorporating the azobenzene moiety were deeply red coloured preventing any further investigation by spectroscopic means due to full beam absorption.

We also investigated thermal switching of the LC as a means for modifying the photonic properties of the composites. The hybrid films of CNMO and 3 show remarkably different dynamic photonic properties than previously reported hybrids with liquid crystalline $8 \mathrm{CB} .^{28}$ In contrast to previous findings, the 3@CNMO hybrids show broad colour changes by variable temperature POM observation between $\sim 70-120^{\circ} \mathrm{C}$, indicating at least two different transitions of the mesogen (Fig. 6a). The first broad transition is related to the transition CR $\leftrightarrow \mathrm{N}_{\mathrm{col}}$ and the second to the $\mathrm{N}_{\text {col }} \leftrightarrow$ ISO phase transition.

To quantify the thermochromic behaviour of the 3@CNMO hybrid, variable-temperature UV-vis and CD spectroscopy were used (Fig. 6b and c and S6 $\dagger$ ). The spectroscopic experiments show a strong and reversible response upon heating and cooling cycles, in good agreement with the variable-temperature POM investigation. Measurements at room temperature or in the isotropic state of the liquid crystalline guest do not show any signal based on the reflectivity of the host material. Even after repeating the heating/cooling cycle, no signal was observed at room temperature. However, a maximum of reflectivity was observed for both datasets (CD and UV-vis spectroscopy) at 90-100 ${ }^{\circ} \mathrm{C}$ and $727 \mathrm{~nm}$ wavelength. The phase transition temperatures observed by spectroscopy of the composite films 


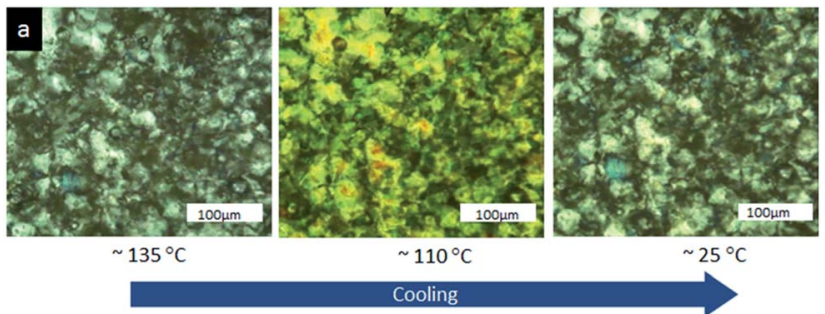

b
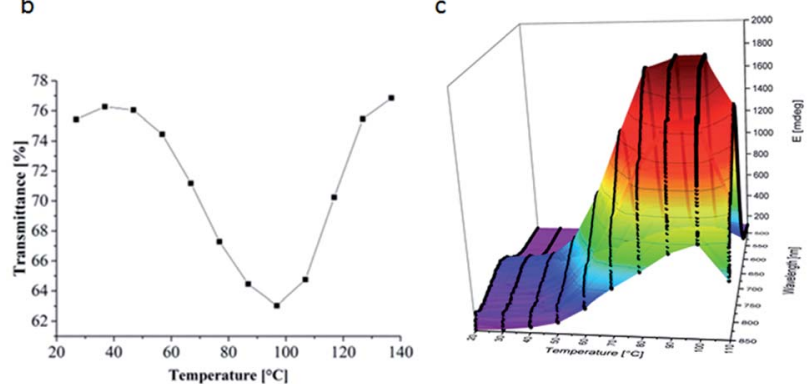

Fig. 6 (a) Variable-temperature POM of 3@CNMO upon heating and cooling cycles; (b) variable-temperature UV-vis spectra of 3 aCNMO upon heating; (c) variable-temperature CD spectra of 3@CNMO upon heating. The UV-vis graph (b) was extracted from the full datasets at $727 \mathrm{~nm}$ at each temperature, corresponding to the maximum in the full spectra. Further spectroscopic data are available in the ESI (Fig. S6†).

differ tremendously from the pristine guest SMLC 3 which can be explained by confinement effects that tend to lower the melting point of substances inside a mesoporous framework compared to the bulk material. ${ }^{9}$ Furthermore, the composite materials differ in that SMLC 3 shows monotropic LC properties upon cooling while the composite materials show the same intermediate behaviour upon cooling and heating. While the bulk material of the SMLC 3 shows distinct signals for each phase transition, the composite shows only a slight increase of the reflectivity that may be traced back to confinement effects. ${ }^{48-51}$

Solid-state NMR experiments were conducted on these novel composite materials; however, they suffer from poor ${ }^{2} \mathrm{H}$ NMR signal-to-noise ratio.

\section{Conclusions}

In summary, we investigated the liquid-crystalline properties of several supramolecular hydrogen-bonded assemblies with phloroglucinol cores and pyridyl-based side chain groups. The cis-trans photoisomerization of the hydrogen-bonded side chains, which is facilitated by UV light irradiation, allows for control over the phase transition from liquid crystalline to isotropic. Introducing different side chain groups, such as azobenzene moieties, proves the versatility of this supramolecular system with respect to tailoring the liquid crystalline properties. Detailed insight into the structural features of the thermotropic liquid crystalline assemblies was obtained from single crystal X-ray crystallography and solid-state NMR studies. The solid-state ${ }^{2} \mathrm{H}$ NMR studies, in particular, shed light on the structure of the $\mathrm{N}_{\text {col }}$ phase.
In addition, infiltration of chiral nematic mesoporous organosilica films with the supramolecular liquid crystal 3 results in a novel temperature-responsive composite material. These systems could be very interesting for future applications in the field of thermochromic or thermoresponsive optical sensors or reflectors. Hence, further investigation of similar composites with less coloured compounds is promising for developing new applications of these materials.

\section{Acknowledgements}

M.G. was generously supported by a postdoctoral fellowship from the German Academic Exchange Service (DAAD). R.Y.D., C.A.M., and M.J.M. thank NSERC for funding (Discovery grants). We thank A. Lam for experimental assistance, and CelluForce Inc. for supplying cellulose nanocrystals.

\section{Notes and references}

1 M. Ramanathan, S. M. Kilbey II, Q. Ji, J. P. Hill and K. Ariga, J. Mater. Chem., 2012, 22, 10389-10405.

2 D. Gentili, F. Valle, C. Albonetti, F. Liscio and M. Cavallini, Acc. Chem. Res., 2014, 47, 2692-2699.

3 J. Maiz, W. Zhao, Y. Gu, J. Lawrence, A. Arbe, A. Alegría, T. Emrick, J. Colmenero, T. P. Russell and C. Mijangos, Polymer, 2014, 55, 4057-4066.

4 J. Siefker, P. Karande and M.-O. Coppens, Expert Opin. Drug Delivery, 2014, 11, 1-13.

5 S. Sanwaria, A. Horechyy, D. Wolf, C.-Y. Chu, H.-L. Chen, P. Formanek, M. Stamm, R. Srivastava and B. Nandan, Angew. Chem., Int. Ed., 2014, 53, 9090-9093.

6 E. Enz, V. La Ferrara and G. Scalia, ACS Nano, 2013, 7, 66276635.

7 S. Sergeyev, W. Pisula and Y. H. Geerts, Chem. Soc. Rev., 2007, 36, 1902-1929.

8 M. Alcoutlabi and G. B. McKenna, J. Phys.: Condens. Matter, 2005, 17, R461-R524.

9 M. Sliwinska-Bartkowiak, J. Gras, R. Sikorski, R. Radhakrishnan, L. Gelb and K. E. Gubbins, Langmuir, 1999, 15, 6060-6069.

10 S. Kubo, Z.-Z. Gu, K. Takahashi, A. Fujishima, H. Segawa and O. Sato, Chem. Mater., 2005, 17, 2298-2309.

11 A. Blanco, E. Chomski, S. Grabtchak, M. Ibisate, S. John, S. Leonard, C. Lopez, F. Meseguer, H. Miguez, J. Mondia, G. Ozin, O. Toader and H. M. van Driel, Nature, 2000, 405, 437-440.

12 C. I. Aguirre, E. Reguera and A. Stein, Adv. Funct. Mater., 2010, 20, 2565-2578.

13 Y. Zhao, Z. Xie, H. Gu, C. Zhu and Z. Gu, Chem. Soc. Rev., 2012, 41, 3297-3317.

14 H. Kitzerow, A. Lorenz and H. Matthias, Phys. Status Solidi, 2007, 204, 3754-3767.

15 H. Kitzerow, A. Lorenz and H. Matthias, Nanophotonic Materials, Wiley-VCH, Weinheim, Germany, 2008.

16 S. Kubo, Z.-Z. Gu, K. Takahashi, A. Fujishima, H. Segawa and O. Sato, J. Am. Chem. Soc., 2004, 126, 8314-8319. 
17 S. Kubo, Z.-Z. Gu, K. Takahashi, Y. Ohko, O. Sato and A. Fujishima, J. Am. Chem. Soc., 2002, 124, 10950-10951.

18 K. Busch and S. John, Phys. Rev. Lett., 1999, 83, 967-970.

19 Y. Shimoda, M. Ozaki and K. Yoshino, Appl. Phys. Lett., 2001, 79, 3627-3629.

20 D. Kang, J. Maclennan, N. Clark, A. Zakhidov and R. Baughman, Phys. Rev. Lett., 2001, 86, 4052-4055.

21 S. W. Leonard, J. P. Mondia, H. M. van Driel, O. Toader and S. John, Phys. Rev. B: Condens. Matter Mater. Phys., 2000, 61, 2389-2392.

22 A. Okabe, T. Fukushima, K. Ariga and T. Aida, Angew. Chem., Int. Ed., 2002, 114, 3564-3567.

23 K. E. Shopsowitz, H. Qi, W. Y. Hamad and M. J. MacLachlan, Nature, 2010, 468, 422-425.

24 K. E. Shopsowitz, W. Y. Hamad and M. J. MacLachlan, J. Am. Chem. Soc., 2012, 134, 867-870.

25 K. E. Shopsowitz, W. Y. Hamad and M. J. MacLachlan, Angew. Chem., Int. Ed., 2011, 50, 10991-10995.

26 F. Reinitzer, Monatsh. Chem., 1888, 421-441.

27 J. A. Kelly, C. P. K. Manchee, S. Cheng, J. M. Ahn, K. E. Shopsowitz, W. Y. Hamad and M. J. MacLachlan, J. Mater. Chem. C, 2014, 2, 5093-5097.

28 M. Giese, J. C. De Witt, K. E. Shopsowitz, A. P. Manning, R. Y. Dong, C. A. Michal, W. Y. Hamad and M. J. MacLachlan, ACS Appl. Mater. Interfaces, 2013, 5, 6854-6859.

29 A. P. Manning, M. Giese, A. S. Terpstra, M. J. MacLachlan, W. Y. Hamad, R. Y. Dong and C. A. Michal, Magn. Reson. Chem., 2014, 52, 532-539.

30 C. A. Michal, K. Broughton and E. Hansen, Rev. Sci. Instrum., 2002, 73, 453-458.

31 C. Koopmans and H. Ritter, J. Am. Chem. Soc., 2007, 129, 3502-3503.

32 Y. Xu, W. Qu, Q. Yang, J. Zheng, Z. Shen, X. Fan and Q. Zhou, Macromolecules, 2012, 45, 2682-2689.

33 L. Cui and Y. Zhao, Chem. Mater., 2004, 16, 2076-2082.

34 Y. Uchida, K. Suzuki and R. Tamura, J. Phys. Chem. B, 2012, 116, 9791-9795.
35 J. H. Lee, M.-J. Han, S. H. Hwang, I. Jang, S. J. Lee, S. H. Yoo, J. Y. Jho and S.-Y. Park, Tetrahedron Lett., 2005, 46, 71437146.

36 M.-C. Chiang and W. H. Hartung, J. Org. Chem., 1945, 10, 2125.

37 U. Kumar, T. Kato and J. M. J. Fréchet, J. Am. Chem. Soc., 1992, 114, 6630-6639.

38 S. J. Lee, M. K. You, S. W. Lee, J. Lee, J. H. Lee and J. Y. Jho, Liq. Cryst., 2011, 38, 1289-1299.

39 L. M. Salonen, M. Ellermann and F. Diederich, Angew. Chem. Int. Ed., 2011, 50, 4808-4842.

40 D. Goldfarb, Z. Luz and H. Zimmermann, J. Phys., 1981, 42, 1303-1311.

41 D. Goldfarb, R. Y. Dong, Z. Luz and H. Zimmermann, Mol. Phys., 1985, 54, 1185-1202.

42 L. Wang, Chem. Phys., 1990, 142, 335-343.

43 L. Lin, Mol. Cryst. Liq. Cryst., 1987, 146, 41-54.

44 J. W. Emsley, Nuclear Magnetic Resonance of Liquid Crystals, D. Reidel Publishing Company, Dordrecht, Boston, Lancaster, 1985.

45 R. Chitra, A. Das, R. R. Choudhury, M. Ramanadham and R. Chidambaram, Pramana, 2004, 63, 263-269.

46 L. S. Batchelder, J. Chem. Phys., 1981, 74, 4791-4799.

47 M. Ricci, R. Berardi and C. Zannoni, Soft Matter, 2008, 4, 2030-2038.

48 M. Alcoutlabi and G. B. McKenna, J. Phys.: Condens. Matter, 2005, 17, R461-R524.

49 R. Guégan, D. Morineau, R. Lefort, W. Béziel, M. Guendouz, L. Noirez, A. Henschel and P. Huber, Eur. Phys. J. E: Soft Matter Biol. Phys., 2008, 26, 261-273.

50 M. Wittebrood, D. Luijendijk, S. Stallinga, T. Rasing and I. Musevic, Phys. Rev. E: Stat. Phys., Plasmas, Fluids, Relat. Interdiscip. Top., 1996, 54, 5232-5234.

51 R. J. Ondris-Crawford, G. P. Crawford, J. W. Doane and S. Zumer, Phys. Rev. E: Stat. Phys., Plasmas, Fluids, Relat. Interdiscip. Top., 1993, 48, 1998-2005. 